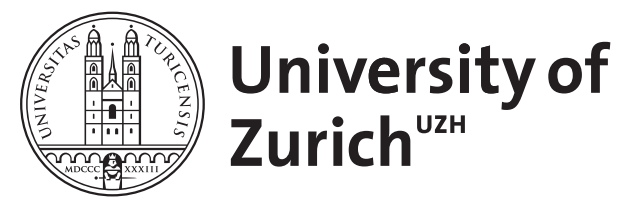

\title{
A New Illusion at Your Elbow
}

\author{
Brugger, $\mathrm{P}$; Meier, R
}

\begin{abstract}
On experiencing distal-proximal tactile motion on the volar side of the forearm starting at the wrist, subjects significantly anticipate touch of the elbow crook. This illusion, popular as a children's game, was quantified in ninety participants (forty-seven women) on both arms. As a top-down explanation of the illusion, we discuss a model of Bayesian inferences. As a bottom-up contribution, we consider afterdischarges of cortical neurons, which receive input from skin mechanoreceptors specifically driven by slow-motion tactile stimuli. Like previously described illusions, the elbow crook illusion is larger on the nondominant arm. Women showed a smaller illusion than men, giving testimony to their reportedly superior cutaneous sensitivity.
\end{abstract}

DOI: https://doi.org/10.1068/p7910

Posted at the Zurich Open Repository and Archive, University of Zurich ZORA URL: https://doi.org/10.5167/uzh-115488

Journal Article

Accepted Version

Originally published at:

Brugger, P; Meier, R (2015). A New Illusion at Your Elbow. Perception, 44(2):219-221.

DOI: https://doi.org/10.1068/p7910 


\title{
A new illusion at your elbow
}

\author{
Peter Brugger and Rebekka Meier \\ Neuropsychology Unit \\ Dept. of Neurology \\ University Hospital Zurich
}

\begin{abstract}
On experiencing distal-proximal tactile motion on the volar side of the forearm starting at the wrist, subjects significantly anticipate touch of the elbow crook. This illusion, popular as a children's game, was quantified in 90 participants (47 women) on both arms. The illusion is explained by afterdischarges of those $\mathrm{S} 1$ neurons, which receive input from skin mechanoreceptors specifically driven by slow-motion $(<5 \mathrm{~cm} / \mathrm{s})$ tactile stimuli. As previously described illusions, it is larger on the nondominant arm. Women showed a smaller illusion than men, giving testimony to their reportedly superior cutaneous sensitivity.
\end{abstract}

Among the manifold illusions of the cutaneous sense (Lederman and Jones, 2011), some are not readily amenable to playful experimentation as they require substantial technical equipment (Hayward, 2008). One exception is a game, Swiss children typically enjoy on playgrounds. They stimulate the inner side of a friend's forearm by slowly moving a finger from the wrist towards the crook of the elbow. Eyes closed, the friend has to shout "stop!" on feeling the crook being reached. On opening the eyes, there is much amazement about an anticipation error, frequently in the order of several centimeters. We investigated the crookof-the-elbow illusion under controlled conditions (Fig. 1) and suggest a neurophysiological explanation of its origin.

****************** INSERT FIG. 1 ABOUT HERE $* * * * * * * * * * * * * * * * * * *$

Our measurements confirm a powerful and robust illusory anticipation of touch at the elbow crook when the tactile stimulus is slowly moved in a proximal direction starting at the wrist. In other words, the track on the skin appears subjectively enlarged. This is in contrast to most previously described cutaneous motion illusions that consist in a subjective length contraction. These have been accounted for by a model of Bayesian inference (Goldreich, 2007), which views the most probable cutaneous percept as "a compromise between imprecise sensorineural information and the observer's expectation of slow movement" (Goldreich, 2007, p. 2), arguing that, under natural circumstances, object motion on the skin is slow. In the case of movements faster than those encountered in natural scenarios, as applied in many laboratory situations, Bayesian slow-motion priors would be violated and thus give rise to an illusory length contraction. The cutaneous motion illusion studied here occurs in response to a stimulation velocity at or even below the velocities typically experienced in everyday life (e.g. during caressing movements or the crawling of an insect) and a participant's expectations of a 
low speed of tactile motion are not violated. Top-down effects of expectation on perceptual experience are thus unlikely the source of the elbow-crook illusion. Instead, an explanation may be found in the characteristics of skin mechanoreceptors and the S1 neurons they project to. $\mathrm{C}$ and $\mathrm{A} \delta$ fibers innervating mechanoreceptors are prominently driven by slow moving ( $<$ $5 \mathrm{~cm} / \mathrm{s}$ ) stimuli, and the corresponding neurons in S1 are known for their exceptionally long afterdischarges (Whitsel et al., 1986; McKenna et al., 1984). These afterdischarges were made responsible for the subjective enlargement of cutaneous motion tracks applied at velocities below $5 \mathrm{~cm} / \mathrm{s}$ (Whitsel et al., 1986). These authors had their subjects indicate the offset point of a tactile motion on the dorsal aspect of the forearm and found a subjective overshooting at velocities below $5 \mathrm{~cm} / \mathrm{s}$, but an increasing undershooting with increasing stimulation velocities (between $25 \mathrm{~cm} / \mathrm{s}$ and $250 \mathrm{~cm} / \mathrm{s}$ ). The displacement of the endpoint of a slow-motion track on the skin in the direction of movement may be equivalent to the error of anticipating a body landmark in the case of a continuing motion. The fact that, in the subset of participants stimulated in a proximo-distal direction, the anticipation error was only small may be accounted for by a proximal-distal gradient of increasing tactile sensitivity (Weinstein, 1968) and a locognosic acuity which is especially pronounced around the wrist (Cody et al., 2008). The observation that men showed a stronger illusion than women corroborates previous findings of a better cutaneous sensitivity in female compared to male participants (Weinstein, 1968; Chen et al., 1995; Peters et al., 2009). The laterality effect, i.e. the larger illusory anticipation on the left forearm, was unexpected, as for stationary stimuli, no marked side differences in tactile sensitivity are apparent (Weinstein, 1968). It is in line, however, with a stronger multisensory (visual-tactile-proprioceptive) illusion on the nondominant compared to the dominant hand after dynamic tactile stimulation (slow-motion brushing; Ocklenburg et al., 2011). The more pronounced arm differences in men compared to women may reflect their stronger functional cerebral hemispheric laterality (McGlone, 1980) and the absence of modulating effects of the menstrual cycle (Hausmann, 2005).

Explaining the mechanisms of an illusion should never aim at diminishing our amazement on experiencing it. In this sense, the surprise by the little playground game investigated here will hopefully remain at your elbow.

\section{REFERENCES}

Chen C C, Essick G K, Kelly DG, Young MG, Nestor JM, Masse B 1995 "Gender-dependent, side-dependent and site-dependent variations in human perioral spatial resolution" Archives of Oral Biology 40, 539-548

Cody FW, Garside RA, Lloyd D, Poliakoff E 2008 "Tactile spatial acuity varies with site and axis on the human upper limb" Neuroscience Letters 433, 103-108

Goldreich D 2007 "A Bayesian perceptual model replicates the Cutaneous Rabbit and other tactile spatiotemporal illusions" PLoS ONE 2(3), e333

Hausmann M, 2005 "Hemispheric asymmetry in spatial attention across the menstrual cycle" Neuropsychologia 43, 1559-1567

Hayward V 2008 "A brief taxonomy of tactile illusions and demonstrations that can be done in a hardware store" Brain Research Bulletin 75, 742-752

Ledermann SJ, Jones LA 2011 "Tactile and haptic illusions" IREE Transactions on Haptics 4, 273-394

McGlone J 1980 "Sex differences in human brain asymmetry: a critical surview" Behavioral and Brain Sciences 3, 215-227.

McKenna TM, Light AR, Whitsel BL 1984 "Neurons with unusual response and receptivefield properties in upper laminae of cat S-I cortex" Journal of Neurophysiology 51, 10551076 
Ocklenburg S, Ruther N, Peterburs J, Pinnow M, Gunturkun O 2011 "Laterality in the rubber hand illusion" Laterality 16, 174-167

Peters RM, Hackeman E, Goldreich D 2009 "Diminutive digits discern delicate details: fingertip size and the sex difference in tactile spatial acuity" Journal of Neuroscience 29, 15756-15761

Weinstein S 1968 "Intensive and extensive aspects of tactile sensitivity as a function of body part, sex, and laterality" In Kenshalo DR, The skin senses, Springfield, Charles C Thomas (pp. 195-222)

Whitsel BL, Franzen O, Dreyer DA, Hollins M, Young M, Essick GK, Wong C 1986

"Dependence of subjective traverse length on velocity of moving tactile stimuli" Somatosensory Research 3, 185-196

\section{FIGURE CAPTION}

LEFT: Participant's arm was stretched out and stimulated on the volar side with a blunt stylus moving from the wrist towards the elbow's crook. A constant speed of approx. 2 to $3 \mathrm{~cm} / \mathrm{s}$ had been practiced beforehand, and the constant soft pressure was determined by the stylus' weight $(5 \mathrm{~g})$. Skin stretching was carefully avoided. Eyes closed, the participant had to indicate verbally once touch was experienced in the crook. The deviation, measured to the nearest $\mathrm{mm}$, could not be observed by the participant.

RIGHT: 47 women and 43 men gave written informed consent to participate in the experiment approved by the ethics committee of the university of Basel. They were all righthanded and of comparable age (mean=41.9 yrs., $\mathrm{SD}=16.4$ yrs.). Participants were tested on both left and right arm (counterbalanced order). Overall, the anticipation error was significant (mean $=3.0 \mathrm{~cm}, \mathrm{SD}=2.2 \mathrm{~cm} ; \mathrm{t}=14.1, \mathrm{df}=89, \mathrm{p}<.0001)$. ANOVA revealed a main effect of sex $(\mathrm{F}(1,88)=6.7, \mathrm{p}=.011$ and $\operatorname{arm}(\mathrm{F}(1,88)=11.0, \mathrm{p}=.001)$. Women showed a smaller error than men, and the illusion was larger on the left than the right forearm. The interaction also reached significance $(\mathrm{F}(1,88)=4.3, \mathrm{p}=.042)$; the arm difference being larger in men than in women.

Fifty-eight of the participants (31 women) were also tested in a proximo-distal direction, having to indicate when the moving stimulus approaching from the elbow crook seemed to have reached the wrist. The illusory anticipation of the wrist (mean $=1.4 \mathrm{~cm}, \mathrm{SD}=1.0 \mathrm{~cm}$ ) was significantly smaller than that on the elbow $(t=5.8, d f=57, p<.0001$; data not shown).

\section{ACKNOWLEDGMENTS}

We thank Daniel Goldreich and Gregory Essick for helpful discussions and advice. 
FIGURE 1:
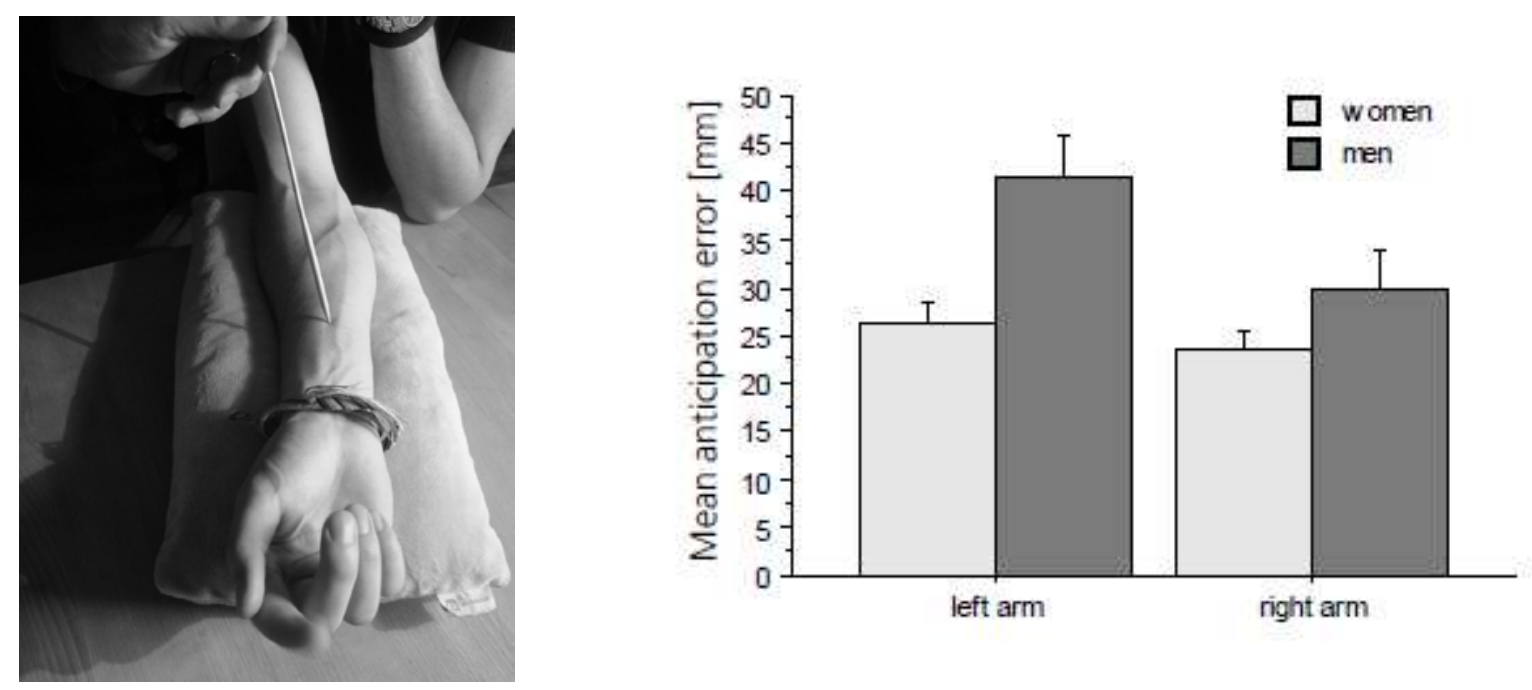